\title{
Z wie Zweitmeinung oder die Folgen von "Psycho»
}

\section{Dominik Heim}

PD Dr. med., Facharzt für Chirurgie, Mitglied FMH, Klinik Hohmad Thun/lexiatrik Luzern

Elegant streicht sie das Duschmittel auf ihre Arme, eine unscharfe, schwarze Gestalt bildet sich am Duschvorhang ab, der Vorhang wird zurückgerissen, ein breites Messer sticht zu, immer und immer wieder, sie kreischt, die $\mathrm{Mu}$ sik setzt mit schrillen Geigen zum Crescendo an, langsam sinkt sie zu Boden, ein lebloses Auge blickt schräg in die Kamera, oben spritzt Wasser aus dem Duschkopf, und unten fliesst das mit Wasser vermischte Blut in den Duschablauf (Szene aus Psycho von Alfred Hitchcock).

Und im biographischen Spielfilm Hitchcock (2012 von Sacha Gervasi) sieht man Hitchcock (Anthony Hopkins) einen Veitstanz im Vestibül des Kinos aufführen, als er die entsetzten Schreie der $\mathrm{Zu}$ schauerinnen und Zuschauer im Kinosaal hört-geschafft!

Diese Duschszene aus Psycho

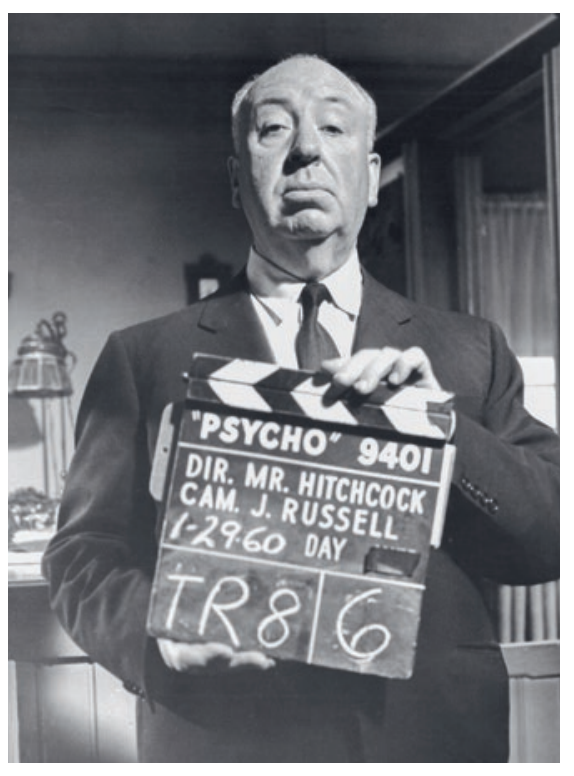

schaft von Roland P. Jakob (1994-1996) Bemühungen, solche Zweitmeinungen für obligatorisch zu erklären [1]. Wenn man die zahlreichen versicherungsmedizinischen Gutachten zu fraglichen Sorgfaltspflichtverletzungen betrachtet, dann kann man dieser Idee viel Positives abgewinnen. In der Tat ist der moderne Patient trotz oder wegen seines GoogleWissens nicht selten nach einem Aufklärungsgespräch verunsichert.

Aber Zweitmeinungen dürfen nicht dazu dienen, die (allenfalls) verunsicherte Patientin als eigene «Kundin» (Ausdruck der Rechtschutzversicherungen) $\mathrm{zu}$ akquirieren. Auch darf zwischen Erstmeinendem und Zweitmeinendem kein gegenseitiges Interesse vorhanden sein. Ja, eine ehrliche Zweitmeinung bedeutet auch, seine eigenen Interessen (1960) gilt bis heute als unerreicht mit ihren präzisen Einstellungen, Schnitten und eben nur angedeuteter Brutalität, hat unzählige Nachahmer im Filmbusiness gefunden und habe den Horrorfilm eigentlich begründet, sagen einige Kritiker. Da bin ich zwar nicht sicher, wenn ich an Nosferatu (F.W. Murnau, 1922) denke. Die Aufnahmen der Duschszene haben eine ganze Woche gedauert, bis der Regisseur mit seinem Hang zur Perfektion zufrieden war. Auch der Erfolg fiel ihm, dem master of suspense, nicht in den Schoss, vielmehr fielen ihm die Filmstudiobosse in den Rücken und verlangten Änderungen, bevor der Film in die Kinos kommen durfte. Und Psycho wurde ein Riesenerfolg! Ob die erste Version von Hitchcock auch ohne diese second opinion der Studiokritiker ein Erfolg geworden wäre, wird man nie wissen.

Bildnachweis sda-keystone

Dominik Heim Bodenweg 25 CH-3714 Frutigen

heim.dominik[at]bluewin.ch

\section{Eigene Interessen hintenanstellen}

Zweitmeinungen prägen zunehmend auch die medizinische Kultur. In der Schweizerischen Orthopädengesellschaft (swiss orthopaedics) gab es in der Präsident- hintanzustellen. Das ist anspruchsvoll. Aber ich bin überzeugt, dass eine second opinion zu einer Reduktion der Operationszahlen und - trotz Kosten für eine Zweitmeinung-zu optimierten Behandlungen führt.

\section{Ob die erste Version von Hitchcock auch ohne}

die second opinion der Kritiker ein Erfolg geworden wäre?

Zweitmeinungen spielen auch in ganz andern Sparten des Lebens eine Rolle: Haben Sie Ihr Bike in einer dieser Grosskonzernen angehörenden Ladenketten gekauft? Und sind Sie vorher in ein versiertes Velogeschäft gegangen für eine zusätzliche Beratung? Darf ich Ihnen eine Zweitmeinung geben? Kaufen Sie ein Velo mit 29-Zoll-Rädern, und lassen Sie sich die carpaltunnelschonenden "Zusatzhörnli» am Lenker montieren - sie werden fahren (fast) wie mit einem E-Bike!

\footnotetext{
Literatur

1 Mariama Kaba. Die Schweizerische Gesellschaft für Orthopädie und Traumatologie seit 1992. Basel; Schwabe Verlag: 2020.
} 